\title{
Observed Area Under the Curve Infinity by Dose
}

National Cancer Institute

\section{Source}

National Cancer Institute. Observed Area Under the Curve Infinity by Dose. NCI

Thesaurus. Code C85762.

The observed area under the curve (AUC) extrapolated to infinity divided by the dose. 